\title{
The second generation of the GFZ Reference Internal Magnetic Model: GRIMM-2
}

\author{
V. Lesur, I. Wardinski, M. Hamoudi, and M. Rother \\ Helmholtz Centre Potsdam, GFZ German Research centre for Geosciences, Telegrafenberg, 14473, Germany \\ (Received December 18, 2009; Revised June 25, 2010; Accepted July 2, 2010; Online published December 31, 2010)
}

\begin{abstract}
We present the second generation of the GFZ Reference Internal Magnetic Model (GRIMM-2), that was derived for the preparation of the GFZ candidate for the 11th generation of the IGRF. The model is built by fitting a vector data set made of CHAMP satellite and observatory data, spanning the period 2001.0 to 2009.5. The data selection technique and the model parametrization are similar to that used for the derivation of the GRIMM model (Lesur et al., 2008). The obtained model is robust over the time span of the data. However, the secular variation above spherical harmonic degree 13 becomes less controlled by the data and is constrained by the applied regularization before 2002 and after 2008.5. At best, only the spherical harmonic degrees 3 to 6 are robustly estimated for the secular acceleration. The problem associated with the first two spherical harmonic degrees of the secular acceleration model arise from the difficulty in separating the core field signal from the external fields and their internally induced counterparts. The regularization technique applied smoothes the magnetic field model in time. This affects all spherical harmonic degrees, but starts to be significant at spherical harmonic degree 5 .
\end{abstract}

Key words: Geomagnetism, core field modeling, IGRF.

\section{Introduction}

The ongoing mission of the CHAMP satellite provides very high quality vector measurements of the Earth's magnetic field which have led in the recent past to numerous studies about its external and internal sources (Reigber et al., 2005). In 2009, the satellite has been revolving in an orbit of very low altitude $(\simeq 320 . \mathrm{km})$. This, combined with the fact that the external magnetic field perturbations were small due to a very long period of low solar activity, serve studies of internal fields. Furthermore, in view of providing the best possible data set for the preparation of the 11th version of the IGRF, fully processed CHAMP vector data have been made available up to 2009.5. This motivate a renewed effort in modeling of the core magnetic field to high spatial and temporal resolution.

In this study we develop the second generation of the GRIMM model, covering the years 2001 to 2009.5. In its prior version (Lesur et al., 2008), the GRIMM model has been built from a data set made of CHAMP satellite data covering years 2001 to 2006 and hourly mean values obtained from 132 geomagnetic observatories. The data selection process was set to optimize the model time resolution. The GRIMM model showed a general agreement in mapping the temporal and spatial characteristics of magnetic field features with the series of CHAOS models (Olsen et al., 2006b, 2009), which were mainly based on satellite data from CHAMP and Ørsted. The data selection criteria

Copyright (c) The Society of Geomagnetism and Earth, Planetary and Space Sciences (SGEPSS); The Seismological Society of Japan; The Volcanological Society of Japan; The Geodetic Society of Japan; The Japanese Society for Planetary Sciences; TERRAPUB.

doi: $10.5047 /$ eps.2010.07.007 are different for both modeling approaches, but they rely on a similar parameterization of the temporal evolution of the magnetic field, and consequently revealed a rapid fluctuation of the secular acceleration. Such detailed and robust description of the secular acceleration of the Earth's magnetic field allows to investigate processes driving the temporal evolution of the field itself such as changes of the flow inside the liquid outer core. Other core magnetic field models are available (e.g. Maus et al., 2006; Thomson and Lesur, 2007) but with a different time parameterization that does not allow a continuous mapping in time of the secular acceleration.

We use the new large CHAMP data set to derive the second generation of the GRIMM model-GRIMM-2, and to derivate our IGRF candidate model. In this work some questions regarding the robustness of secular acceleration estimates are addressed. Although the GRIMM series of models aim to model all aspects of the magnetic field of internal origin, we present here only results concerning the field generated in the Earth's core. An associated model of the lithospheric field is available, but it has been derived independently from the core field model.

The next section is dedicated to briefly describe the data selection techniques, the model parameterization of GRIMM-2, and the model estimation techniques. The modifications introduced for this second generation of the model are highlighted. The third section presents the obtained model which then is discussed in the fourth section. 


\section{Data Set, Data Selection, Model Parameteriza- tion and Model Estimation}

\subsection{Data set, data selection}

The model GRIMM-2 is built from CHAMP satellite magnetic vector data, and observatory hourly mean vector data. The most recent version 51 Level-2 CHAMP satellite data span the epochs 2001.0 to 2009.58 and include improved time dependent FGM-ASC orientation corrections (i.e. orientation of the fluxgate magnetometers relative to the reference frame defined by the star cameras). Observatory hourly mean data are only used up to 2009.0.

The mid- and low-latitude satellite data lie in-between $\pm 55^{\circ}$ magnetic latitudes. From these data, only the $X$ and $Y$ components in Solar-Magnetic (SM) system of coordinates are selected for magnetically quiet times following the criteria:

- Positive value of the $Z$-component of the interplanetary magnetic field (IMF- $B_{Z}$ ) to minimize possible re-connection of the magnetic field lines with the Interplanetary Magnetic Field (IMF).

- $20 \mathrm{~s}$ minimum between sampling points such that the nonmodeled lithospheric field does not generate correlated errors between data points.

- Local time between 23:00 and 05:00, and the sun below the horizon at $100 \mathrm{~km}$ above the Earth's reference radius $(a=6371.2 \mathrm{~km})$, to minimize the contribution from the magnetic field generated in the ionosphere.

- Norm of the Vector Magnetic Disturbances (VMD, Thomson and Lesur, 2007) less than $20 \mathrm{nT}$ and norm of its time derivative less than $100 \mathrm{nT} /$ day.

- High accuracy of the FGM magnetometer readings (quality flag 1 set to 0 ) and dual star-camera mode (quality flag 2 set to 3 ).

- Star camera outputs checked and corrected (Flag digit describing the attitude processing technique larger than 1).

At high latitudes, i.e. outside the $\pm 55^{\circ}$ magnetic latitudes interval, the three component vector magnetic satellite data are used in North, East, Center (NEC) system of coordinates. Their selection criteria differ from those listed above in two points:

- Data are selected at all local time, and independently of the sun position.

- Data sampled in single-camera mode are used.

These two selection criteria were chosen to avoid significant gaps in the time series of high-latitudes data. The second of these points has been introduced specifically for GRIMM-2, but it is associated with only a relatively small amount of data (see Table 1 below) and the overall effect on the final model is small.

The same selection criteria as for mid and low-latitudes satellite data are applied to hourly mean data of geomagnetic observatories (downloaded from the Word Data Center (British Geological Survey-BGS, Edinburgh)). From the 148 observatories with available data for the epochs of interest, the data of 18 observatories were rejected, because of a strong contamination with instrumental noise, base-line jumps and drifts. We point out that we are using hourly mean data, without further processing.

The data selection criteria used here are very similar to those used in the derivation of the first generation of GRIMM and have proven to lead to robust and accurate core field models.

\subsection{Model parameterization}

The model parameterization has been simplified compared to the GRIMM model. We do not attempt here to estimate the toroidal magnetic field generated by the field aligned currents, nor the field generated in the ionosphere at high latitudes. It has been found during the derivation of GRIMM that modeling these contributions only marginally improve the fit to the data, mainly because the temporal parameterization does not provide a useful description of the variations caused by processes in high latitude ionosphere and field aligned currents. Furthermore, co-estimating these magnetic fields and the core magnetic field carries the risk that part of the core field is explained in terms of these external fields and vice versa (Lesur et al., 2008). Also, the lithospheric field is only co-estimated up to Spherical Harmonic (SH) degree 30 together with the other components of the field. Apart from the lithospheric field, the model includes the core field, a representation of the large scale external fields and their associated internally induced counterparts. The crustal offsets at observatory locations are also estimated.

The core field $\mathbf{B}_{c}$ is modeled as the gradient of an internal potential field given as a series of spherical harmonics:

$$
\begin{aligned}
& \mathbf{B}_{c}=-\nabla V_{c}(\theta, \phi, r, t) \\
& V_{c}(\theta, \phi, r, t)=c \sum_{l=1}^{L_{c}} \sum_{m=-l}^{l}\left(\frac{c}{r}\right)^{l+1} g_{l}^{m}(t) Y_{l}^{m}(\theta, \phi) \\
& g_{l}^{m}(t)=\sum_{i=1}^{N_{t}} g_{l i}^{m} \psi_{i}^{6}(t)
\end{aligned}
$$

where $(\theta, \phi, r, t)$ are the colatitude, longitude, radius and time, respectively. The Earth's core reference radius is $c=$ $3485 \mathrm{~km}$, and $Y_{l}^{m}(\theta, \phi)$ are the Schmidt semi-normalized SHs of degree $l$ and order $m$. We use the convention that negative orders, $m<0$, are associated with $\sin (|m| \phi)$ terms whereas null or positive orders, $m \geq 0$, are associated with $\cos (m \phi)$ terms. The maximum SH degree $L_{c}$ for the core field model is set to $L_{c}=16$ even if it is clear that at such high SH degrees, the contribution of the lithospheric field is significant. The Gauss coefficients $g_{l}^{m}(t)$ are time dependent and are represented as a series of $N_{t}$ B-splines, $\psi_{i}^{6}(t)$, of order 6 , with knots one year apart spanning 20002011. This spline order is set to higher value than for GRIMM, such that it is consistent with time smoothing constraints that are presented below.

We consider that the field generated in the lithosphere is not dependent on time. It is defined by:

$$
\begin{aligned}
& \mathbf{B}_{l}=-\nabla V_{l}(\theta, \phi, r) \\
& V_{l}(\theta, \phi, r)=a \sum_{l=L_{c}+1}^{L_{l}} \sum_{m=-l}^{l}\left(\frac{a}{r}\right)^{l+1} g_{l}^{m} Y_{l}^{m}(\theta, \phi)
\end{aligned}
$$

where $a=6371.2 \mathrm{~km}$ is the Earth's reference radius. The lithospheric model maximum SH degree is set to $L_{l}=30$. As the satellite altitude decreased from roughly $480 \mathrm{~km}$ in 
2001 to $310 \mathrm{~km}$ in 2009 , the strength of the short wavelength magnetic field generated in the lithosphere increased. While testing modeling schemes using the End-to-End synthetic data set for the preparation of the Swarm-Mission (Olsen et al., 2006a), it has been revealed that this slowly increasing lithospheric field contribution leaks into the secular variation (SV) model. In order to avoid this aliasing effect, the GRIMM lithospheric field model from SH degree 17 to degree 80 is subtracted from the data in a preprocessing phase. The present modeling effectively corresponds to a correction of the original lithospheric field model of GRIMM, rather than the lithospheric field itself.

The large scale external fields are described by:

$$
\begin{aligned}
& \mathbf{B}_{e}=-\nabla\left(V_{e}(\theta, \phi, r, t)+V_{\mathrm{vmd}}(\theta, \phi, r, t)\right) \\
& V_{e}(\theta, \phi, r, t)=a \sum_{l=1}^{L_{e}} \sum_{m=-l}^{l}\left(\frac{r}{a}\right)^{l} q_{l}^{m}(t) Y_{l}^{m}(\theta, \phi) \\
& q_{l}^{m}(t)=\sum_{i=1}^{N_{t}^{e}} q_{l i}^{m} \psi_{i}^{2}(t) \\
& V_{\mathrm{vmd}}(\theta, \phi, r, t)=a \sum_{m=-1}^{1} \sum_{i=1}^{N_{t}^{e}-1}\left\{\left(\frac{a}{r}\right)^{2} q_{i, m}^{\mathrm{vi}} \mathrm{vmdi}_{i, m}\right. \\
& \left.+\left(\frac{r}{a}\right) q_{i, m}^{\mathrm{ve}} \mathrm{vmde}_{i, m}\right\} Y_{1}^{m}(\theta, \phi)
\end{aligned}
$$

The maximum SH degree for the potential $V_{e}$ is $L_{e}=2$. The temporal variations of the external Gauss coefficients $q_{l}^{m}(t)$ are defined by $N_{t}^{e}$ B-splines, $\psi_{i}^{2}(t)$, of order 2 , corresponding to a piecewise linear representation in between knots. These knots are separated by 3 months, which is consistent with the definition of the VMD index (Thomson and Lesur, 2007). The rapid external field variations and their associated induced counterparts, are described by the VMD index that provides estimates of the large scale magnetic disturbances every 20 minutes. The external and internal part of the VMD index, $\operatorname{vmde}_{i, m}$ and $\mathrm{vmdi}_{i, m}$ respectively, are scaled for a three month period by their associated coefficients, $q_{i, m}^{\mathrm{ve}}, q_{i, m}^{\mathrm{vi}}$. Here, it is not attempted to separate the different contributions to the large scale external field, as it is not clear to which amount the ring current, tail currents, field aligned currents and other sources contribute to this field. This separation may not be possible. In particular, there is no information available about some significant parts of this large scale field at mid- and low-latitude because the data are selected along the $X$ and $Y$ SM direction only (Lesur et al., 2008). Further, the level of noise for high latitude data is very large and the $V_{\mathrm{vmd}}(\theta, \phi, r, t)$ coefficients are not adjusted to fit these data. This way, we allow for a better description of the external field at mid latitudes which, ultimately, leads to a better core field model quality.

\subsection{Model estimation}

In order to obtain a robust core field model for the entire data time span, constraints have to be applied on the model parameters. As we will see below, the spatial complexity of the obtained SV model is not robust for SH degrees higher than 13 . Normally constraints should be applied to control the spatial complexity in order to have an acceptable model at high SH degrees. Because of the fact that the modeled $\mathrm{SV}$ at high $\mathrm{SH}$ degrees shows reasonable behavior in some regions, i.e. mid latitudinal regions, we abandoned the idea of using a spatial constraint in the derivation of GRIMM-2.

In the present work, we concentrate in deriving a model providing an accurate description of the temporal variations up to SH degree 13 and applied two different constraints. First we minimize together with the misfit to the data a measure of the roughness in time of the model:

$$
\Phi_{t 3}=\lambda_{t 3} \int_{\mathcal{T}} \int_{\Omega_{c}}\left|\partial_{t}^{3} B_{r}\right|^{2} d \omega d t
$$

where $\mathcal{T}$ is the model time span 2000-2011, $\Omega_{c}$ the spherical surface with radius $c$ and $B_{r}$ the radial component of the magnetic field. We also minimize a measure of magnetic field acceleration at epochs 2000.0 and 2011.0:

$$
\Phi_{t 2}(t)=\lambda_{t 2} \int_{\Omega_{c}}\left|\partial_{t}^{2} B_{r}\right|^{2} d \omega .
$$

The second temporal constraint becomes necessary, as the SV and acceleration are not fully controlled at the endpoints of the time span by the first constraint. The damping parameters $\lambda_{t 3}$ and $\lambda_{t 2}$ are estimated by comparing the fit to the data and the roughness of the obtained model. The regularization technique applied here is slightly different from the one used in the first generation of GRIMM. Here, all constraints are applied at the core mantle boundary. Finally, we note that using order six B-splines in the time for the parameterization of the core field (see Eq. (1)) is consistent with the third time derivative constraint defined in Eq. (4). In fact the order six B-splines are the "natural" interpolating functions with minimal third time derivative.

The model estimation is made in three successive steps. After computing data densities on a quasi-regular triangular mesh, a first rough model is obtained by a least-square fit, where a satellite datum is weighted depending on the density of the triangular cell it belongs to. The resulting fit to the data is given in Table 1. In the second step, the least-square fit to the data is made using as weights the inverse of the error variances estimated in the first run. We did not introduced a specific treatment to handle the anisotropic error of single-camera mode data because their errors are dominated by the un-modeled contributions of the magnetic field generated by field aligned currents. As the problem is linear, a large range of damping parameter can be investigated rapidly. Once acceptable values of the damping parameters are set, the model is further improved by five runs done using a re-weighted least-squares algorithm with an $L_{1}$ measure of the misfit. These runs are computationally demanding because the set of normal equations has to be re-calculated each time. The starting model used is the output of the second step. By doing only five iterations, the iterative process is not fully converged, but we verified that the part of the model associated with the core field does not vary significantly if further iterations are made.

\section{Results}

The data selection presented in Section 2.1, combined with a model parameterization described in Section 2.2 leads through the processing scheme of Section 2.3 to a description of the core field evolution from 2001.0 to 2009.5. The choice of damping parameter was done by evaluating 
Table 1. The residual mean (M) and standard deviation (SD) for all data types in nT, where Sat. and Obs. stand for satellite and observatory, respectively. HL corresponds to high latitudes and SM to the SM coordinate system.

\begin{tabular}{|c|c|c|c|c|c|}
\hline \multirow[t]{2}{*}{ Data types } & \multirow[t]{2}{*}{ Camera mode } & \multirow[t]{2}{*}{ Number of data } & \multirow{2}{*}{$\begin{array}{c}\text { First run } \\
\text { SD }\end{array}$} & \multicolumn{2}{|c|}{ GRIMM-2 } \\
\hline & & & & M & $\mathrm{SD}$ \\
\hline Sat. $X$ (SM) & Dual & 568361 & 3.7 & 0.04 & 2.69 \\
\hline Sat. $Y$ (SM) & Dual & 568361 & 3.9 & -0.43 & 3.15 \\
\hline Sat. $X$ (HL) & Dual & 1100140 & 48.2 & 1.37 & 44.25 \\
\hline Sat. $Y$ (HL) & Dual & 1100140 & 53.8 & 0.44 & 49.51 \\
\hline Sat. Z (HL) & Dual & 1100140 & 20.2 & -0.91 & 17.98 \\
\hline Sat. $X$ (HL) & Single & 332643 & 62.8 & -6.05 & 59.81 \\
\hline Sat. $Y$ (HL) & Single & 332643 & 73.3 & -0.35 & 69.81 \\
\hline Sat. $Z$ (HL) & Single & 332643 & 26.7 & -1.51 & 24.64 \\
\hline Obs. $X(\mathrm{SM})$ & - & 345446 & 3.3 & 0.06 & 3.32 \\
\hline Obs. $Y(\mathrm{SM})$ & - & 345446 & 3.5 & 0.02 & 3.45 \\
\hline Obs. $X$ (HL) & - & 102695 & 19.7 & -1.38 & 19.13 \\
\hline Obs. $Y$ (HL) & - & 102695 & 11.3 & 0.05 & 11.14 \\
\hline Obs. Z (HL) & - & 102695 & 17.3 & 0.22 & 16.98 \\
\hline
\end{tabular}

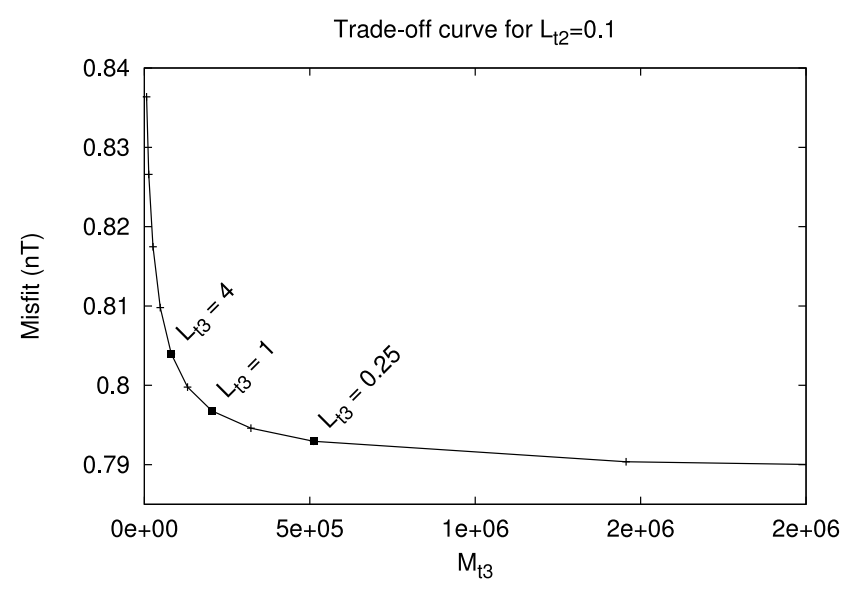

Fig. 1. Trade-off curve between fit to the data and the measure $M_{t 3}$, for $\lambda_{t 2}=0.1$ and several values of $\lambda_{t 3}$ in between $210^{-3}$ and 64 .

systematically model solutions for a large range of parameter values. These model solutions were obtained assuming an $L_{2}$ measure of the misfit. The trade-off curve, i.e. variation of the fit to the data as a function of the measure $M_{t 3}=\frac{\Phi_{t 3}}{\lambda_{t 3}}$, is shown in Fig. 1. The choice of the damping parameter $\lambda_{t 3}=1.0$ close to the knee of the tradeoff curve, corresponds to a rough $L_{2}$-norm solution, that gets much smoother after the re-weighted least-squares iterations. Only solutions for a few parameter values were evaluated under $L_{1}$ measure of the misfit because of the computation time required. The chosen damping parameters are $\lambda_{t 2}=0.1, \lambda_{t 3}=1.0$ and the obtained final fit to the data are given in Table 1 . The residual means (M) are non-zero, even for observatory components where the crustal offsets normally adjust such that the mean is always zero. However, these deviations from zero are due to the use of the $L_{1}$ measure of the misfit (whereas zero means are associated with an $L_{2}$ measure of the misfit). Both means and standard deviations (SD) are particularly large in highlatitude satellite and observatory data. They are associated

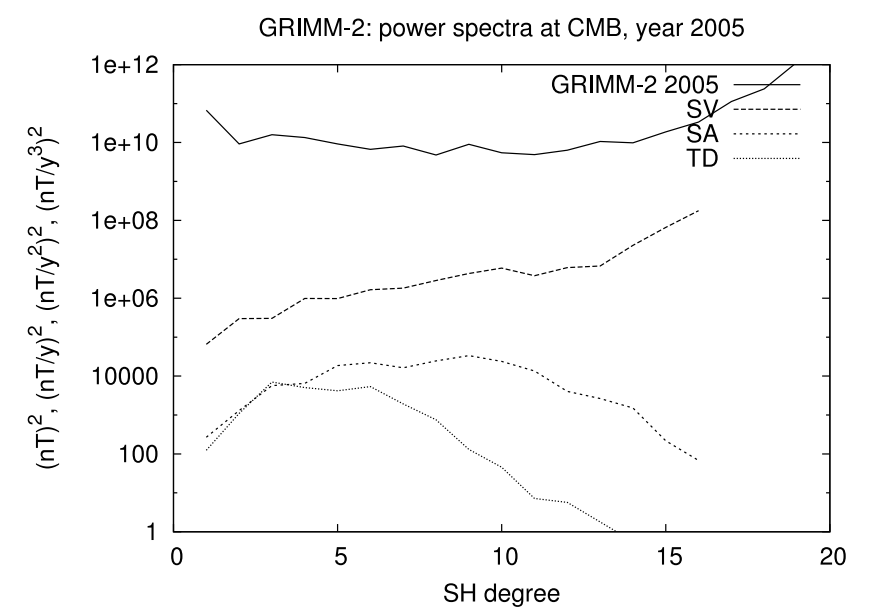

Fig. 2. Power spectra of the core magnetic field and its three time derivatives, calculated at the CMB for year 2005.

with the contributions of non-potential fields, such as those generated by field-aligned currents.

Figure 2 presents the power spectra of a snap-shot of the core field for year 2005.0, its SV, Secular Acceleration (SA) and third time derivative (TD). The spectra are calculated at the core reference radius $c=3485 \mathrm{~km}$. The static core field has a nearly flat spectrum for SH degrees larger than 1 , then it starts growing from SH degree 14, maybe even earlier, because of the contribution from the lithosphere. The SV spectrum has the usual increasing shape, but it also starts to be unstable from $\mathrm{SH}$ degree 14, with a rapid growing rate. We note that the SV spectrum does not converge at the CMB. Both SA and third time derivative spectra are convergent, but this results from the applied damping. The SA itself is constrained to be small only at the model end points, but, because we impose the third time derivative to be small at any time, this affects the whole model span. We note that the third time derivative spectrum reaches large values for low SH degrees-e.g. as large as $710^{3}\left(\mathrm{nT} / \mathrm{y}^{3}\right)^{2}$ 

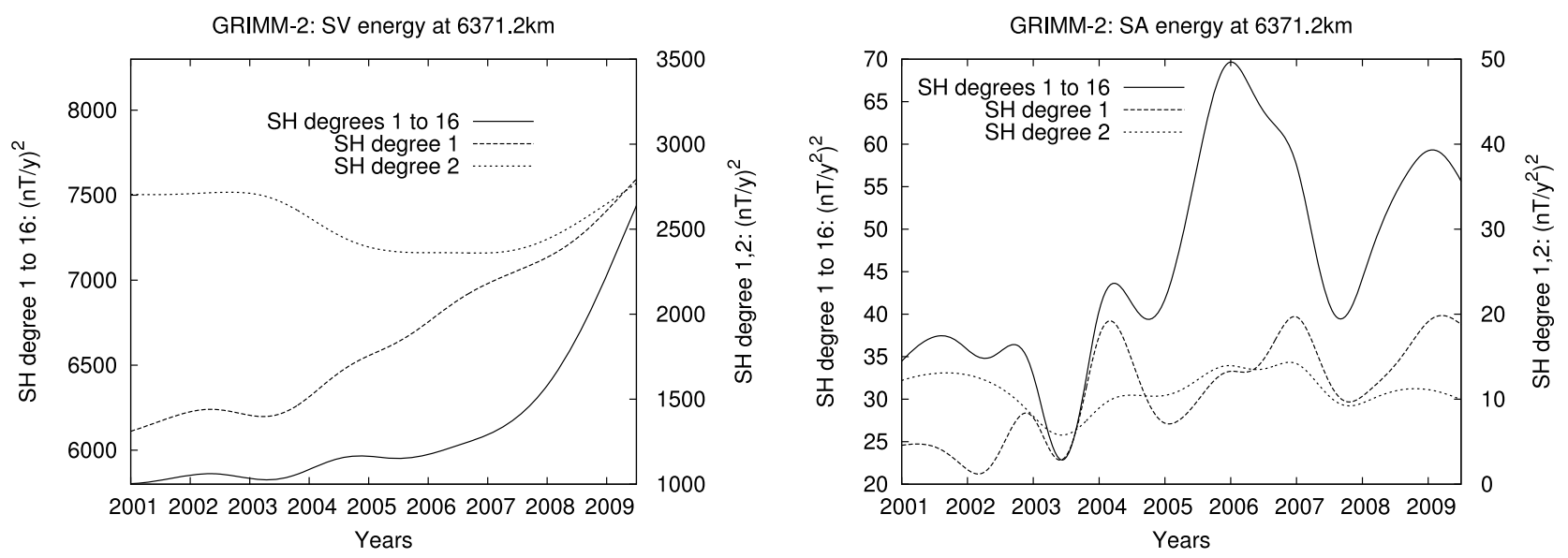

Fig. 3. Spectra of the SV and SA as a function of time.
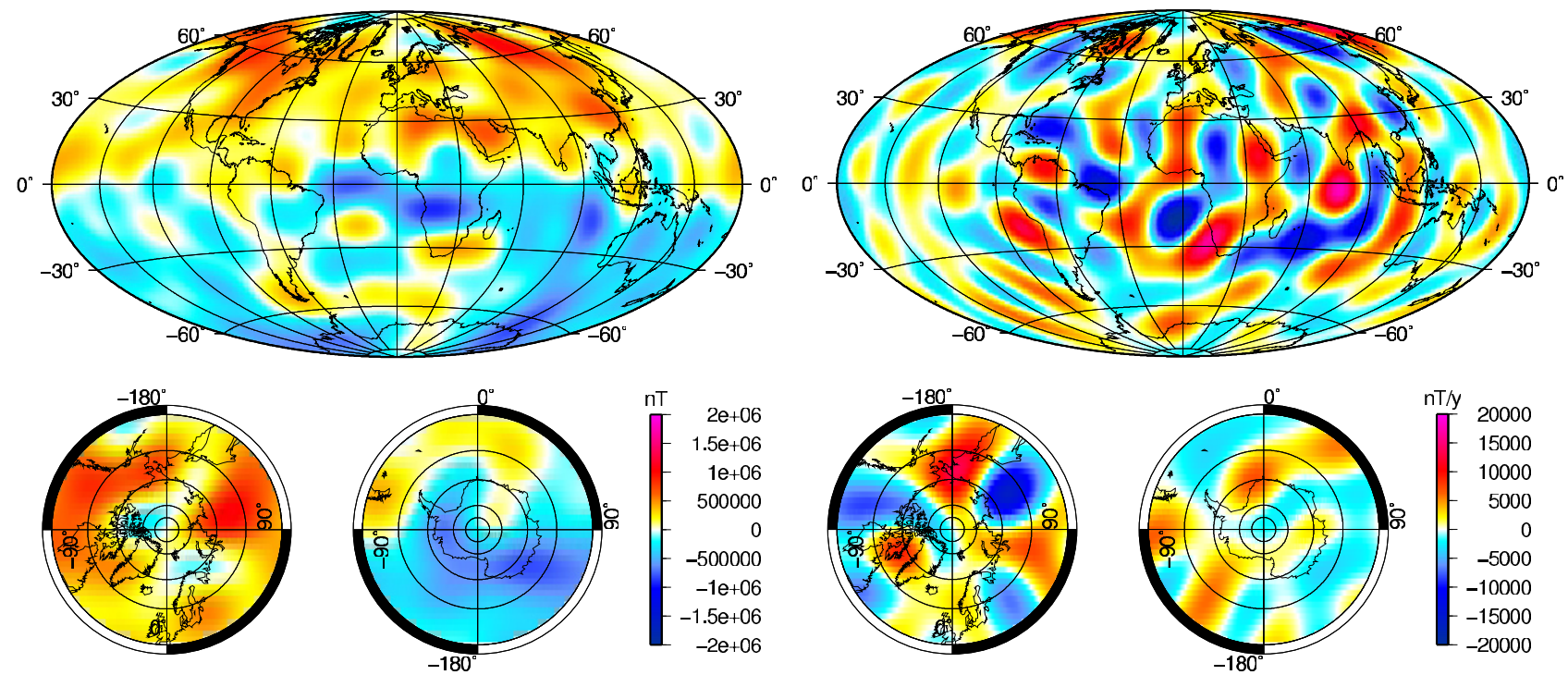

Fig. 4. Vertical down component of the core field and its SV for year 2005, at the CMB for a model truncated to SH degree 13 .

for $\mathrm{SH}$ degree 3. Therefore the SA varies very rapidly for the $\mathrm{SH}$ degrees 1 to degree 4 or 5 . There is no reason to assume that this is not the case at higher $\mathrm{SH}$ degrees, however, the third time derivative is then not resolved by the data and the applied constraints impose a rapidly decreasing spectrum to the magnetic field third time derivative.

These energy spectra evolve in time. In Fig. 3 we present the temporal variation of the SV and SA energies at the Earth's reference radius $a=6371.2 \mathrm{~km}$. At the Earth's surface the SV and SA energies are strongly dominated by the long wave-lengths of the model. The strong increase of the order of $25 \%$ of SV energy from 2007 onward, is due to the first SH degrees. At the core surface this behavior corresponds to a small change because the short wave-lengths are dominant. The SA itself presents a varying energy spectrum, with a maximum at 2006. Most of the rapid temporal oscillations are due to the SH degree $l=1$, but the main contribution to this maximum comes from the $\mathrm{SH}$ degree 3 (not shown). The robustness of these features are discussed in next section.

Figure 4 shows the map of the vertical down component of core field at the CMB for the year 2005. The field morphology is similar to previously published maps for the satellite era, which includes the large reverse flux patch in the southern hemisphere and the sinuous magnetic equator line. Correspondingly, the map of SV radial component for 2005 is comparable to what has been presented previously. However, we note that the spectrum of the SV does not converge at the CMB, and therefore this "image" of the SV may be totally different if a different truncation degree of its $\mathrm{SH}$ series is used.

The robust estimation of SA is one of the main progress in core field modeling these last years. In Lesur et al. (2008) the SA was mapped from 2002 to 2005.5 because the data set did not extend after 2006.7. In Fig. 5 the SA is mapped every year from 2002 to 2009 . The mapping is possible from 2001 to 2009.5 but we do not regard the SA model for these early and late epochs as robust enough. From 2002 to 2009, most of the SA evolution mainly remains below the Indian and South-Atlantic oceans. During the year 2006, the modeled SA reaches absolute values as large as $25 \mathrm{nT} / \mathrm{y}^{2}$ below the South Atlantic. 

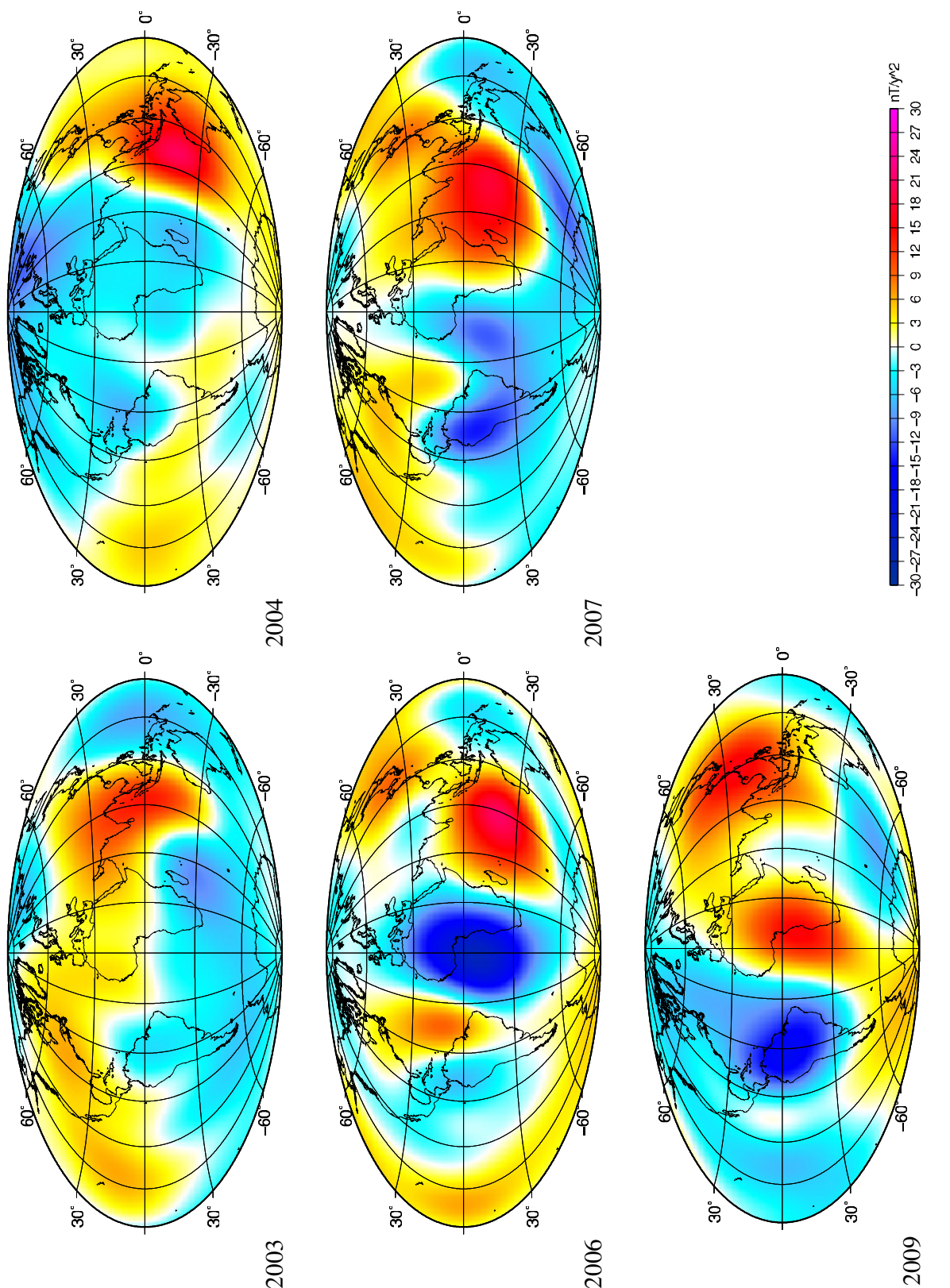

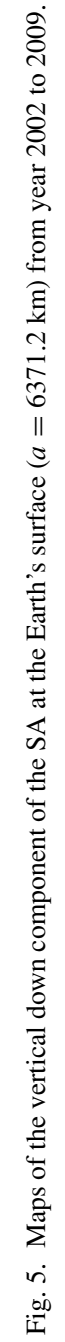
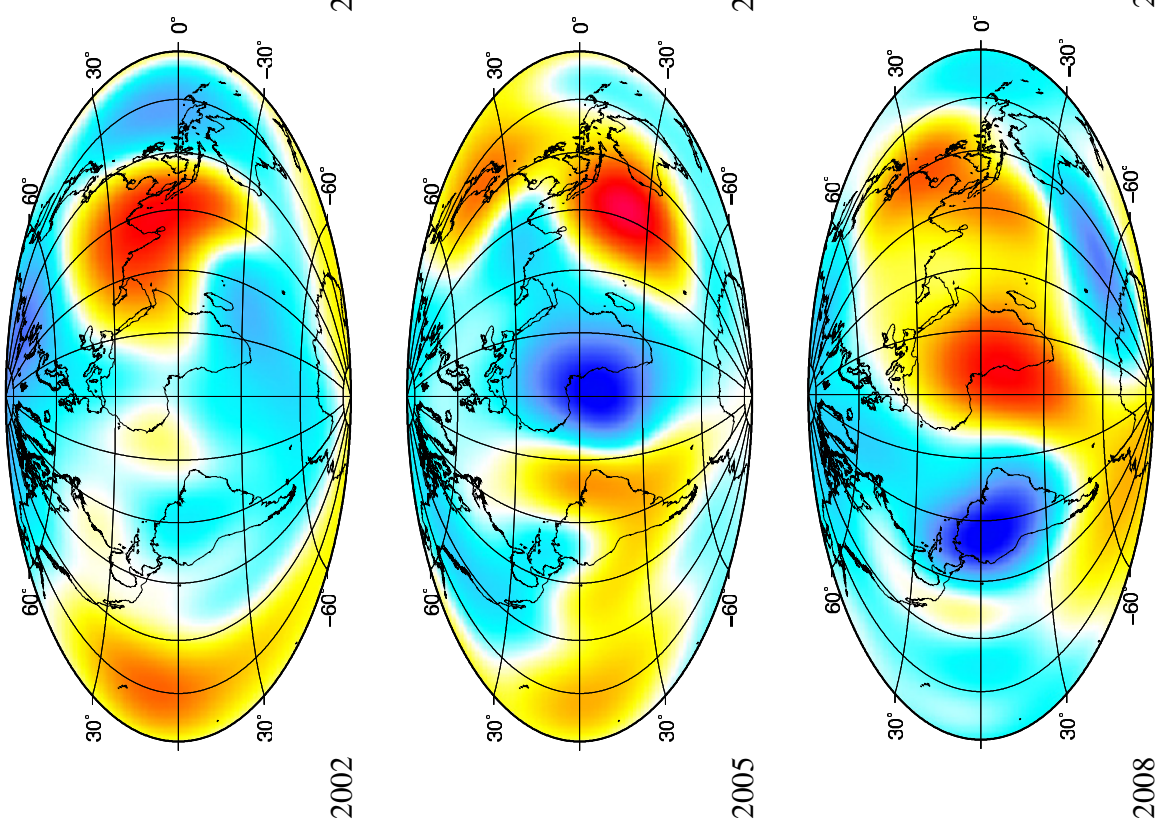

$\stackrel{\infty}{8}$ 

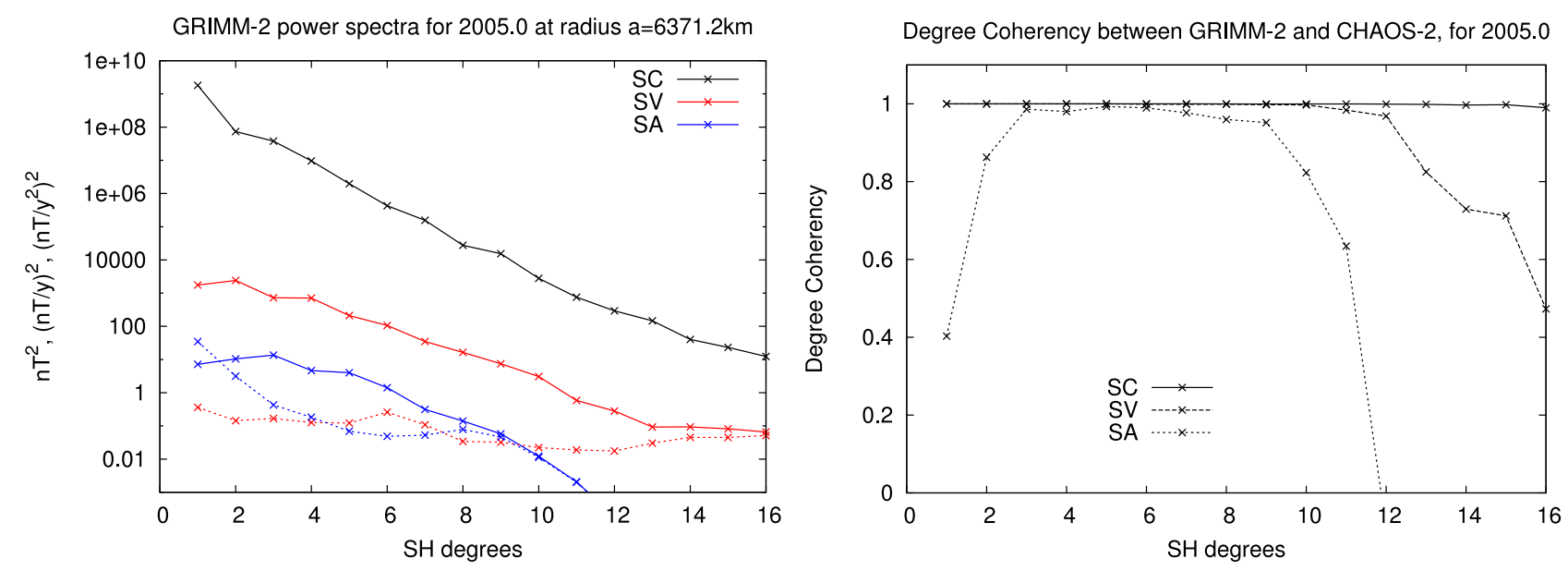

Fig. 6. Comparison between GRIMM-2 and CHAOS-2: Left, power spectra of GRIMM-2 Static Core (SC) field, SV and SA at the Earth's reference radius for 2005.0. The power spectra of the differences relative to CHAOS-2s are shown as doted lines. Right, the degree coherency between GRIMM-2 and CHAOS-2s is displayed.

\section{Discussion}

The GRIMM-2 model was presented in the previous sections. We now turn to the problem of estimating the robustness of the model. In particular it is compared with CHAOS-2s (Olsen et al., 2009), a recent model covering nearly the same time span.

Figure 6 represents the power spectra of GRIMM-2 for year 2005.0 at the Earth's reference radius, and the power spectra of the SV and SA differences relative to CHAOS-2s. The power spectrum of the static field differences is not presented and varies around $2 \mathrm{nT}^{2}$. Regarding the SV estimate, one can see that the power of differences relative to CHAOS-2s stays below $1(\mathrm{nT} / \mathrm{y})^{2}$ for all $\mathrm{SH}$ degrees. The $\mathrm{SV}$ models are therefore in good agreement for the epoch 2005.0 at least up to SH degree 12 maybe 13. This is confirmed by a degree coherency higher than 0.8 up to $\mathrm{SH}$ degree 13. The results for the SA are rather different. Both SA models agree well from SH degree 3 to 6 or 7 , but are different for the longest wave-lengths (SH degree 1 and 2). These differences are directly linked to the modelers estimated data errors. In the present work, we do not regard the rapid temporal variations of the observed field as originating in the core and therefore we do not attempt to fit these variations. It follows that the obtained model is much smoother in time than CHAOS-2s. However, the GRIMM-2 SA model is rough enough such that it fits the data to the level we expect. The temporal variations of the Gauss coefficients we obtained lead to the SA variations mapped in Fig. 5. Again, the degree coherency calculated for the SA (see Fig. 6) shows that there is little agreement on the temporal second derivative of SH degree 1 and 2 Gauss coefficients.

In Fig. 7 are shown the temporal behaviors of the SV Gauss coefficients: $\dot{g}_{1}^{0}(t), \dot{g}_{1}^{1}(t), \dot{h}_{1}^{1}(t), \dot{g}_{3}^{0}(t), \dot{h}_{3}^{3}(t), \dot{g}_{5}^{0}(t)$. These are given with their formal error-bars. Also, the SV Gauss coefficients obtained for an un-constrained model are plotted. For deriving this model, we have solved the inverse problem by calculating the eigenvectors and eigenvalues of the linear system of equations, rejecting all (exact) zero eigenvalues and associated eigenvectors. The ratio of the largest and smallest non-zero eigenvalues was below $10^{12}$. We first see that the temporally un-constrained solution is very close to the constrained one and therefore, the combination of our data selection and model parameterization, independently of the chosen constraints, does not allow a temporal variability of the Gauss coefficients as large as CHAOS-2s variability. The effect of regularization becomes particularly visible at both ends of the time span of GRIMM-2. It is clear that it is the applied regularization that controls the SV before 2002.0 and after 2008.5.

The effect of the constraints is seen on the SA spectrum from $\mathrm{SH}$ degree 7 . At $\mathrm{SH}$ degrees higher than 8 the SA of the un-constrained model is unrealistically large and the obtained SV model oscillates in time around the average value given by the constrained model.

The error-bars shown in Fig. 7 are known to be underestimated (Lowes and Olsen, 2004) and furthermore the model inversion process is regularized. However, they show that $\dot{g}_{1}^{0}(t)$ is much less constrained than coefficients at higher $\mathrm{SH}$ degrees. By a close inspection, one can see that its formal standard deviation estimates have an annual periodicity due to external field contributions to the magnetic data. It is not clear if the observed rapid variation of the $\dot{g}_{1}^{0}(t)$ is due to a signal coming from the core or if it originates in the external fields or its induced counterpart. As seen in Fig. 3, these rapid oscillations of the $\dot{g}_{1}^{0}(t)$ contributes significantly to the temporal variation of the SA energy, but they do not affect significantly the patterns mapped in Fig. 5.

The first temporal derivatives of the other Gauss coefficients present clear and sharp changes in their temporal evolution, generally around 2006 and 2007. These changes are well above the noise level as it could be estimated from the error-bars. We particularly point out the change of slope of the $\dot{h}_{3}^{3}(t)$ just before 2008 that set some challenges for the prediction of the SV over the coming years, and the strong increase of absolute value of $\dot{h}_{1}^{1}(t)$ coefficient. This increase from 2006 is the main contribution to the increase in SV energy already observed in Fig. 3.

In order to better understand the effect of the regulariza- 

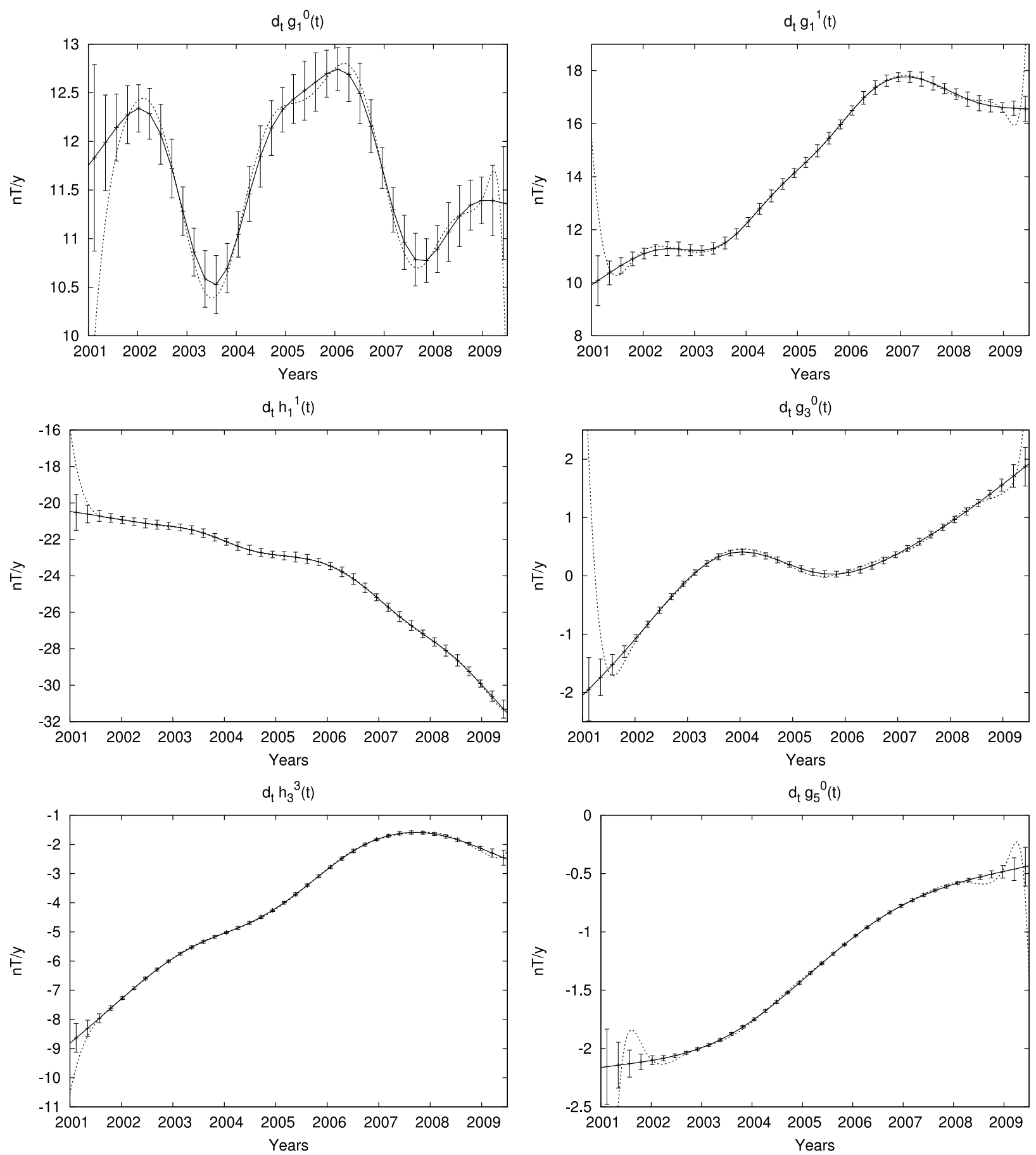

Fig. 7. The evolution of some Gauss coefficient time derivatives, with their formal error-bars, and the same coefficient derivatives obtained when the model is temporally un-constrained.

tion on the resulting model, we conduct a temporal resolution analysis similar to Olsen et al. (2009). The time dependence of the Gauss coefficients is determined by Eq. (1) that we rewrite here:

$$
g_{l}^{m}(t)=\sum_{i=1}^{N_{t}} g_{l i}^{m} \psi_{i}^{6}(t) .
$$

In this analysis, it is assumed that the evolution of each Gauss coefficient is given by a single spline basis function, e.g. $g_{l 8}^{m}$. This spline function is centered in 2005 and we refer to it as the $8^{\text {th }}$ spline function. Figure 8 (left panel) shows the $8^{\text {th }}$ spline function as it is used in our parameterization and how it is modified by the damping for three Gauss coefficients, i.e. $g_{3}^{0}, g_{5}^{0}, g_{8}^{0}$. The filtered function for $g_{1}^{0}$ (not shown) is not significantly different from the original $8^{\text {th }}$ spline function. The effect of the regularization is weak for $g_{3}^{0}$, but starts to be significant for $g_{5}^{0}$ and is large for $g_{8}^{0}$. There is only a weak dependency of these filtered functions with the SH order. This is expected as our regularization measures are independent of the SH order. Such dependency could therefore only be introduced by the data quality or data coverage. It is clear that at SH degrees higher than 8 most of the time resolution of the model vanishes 

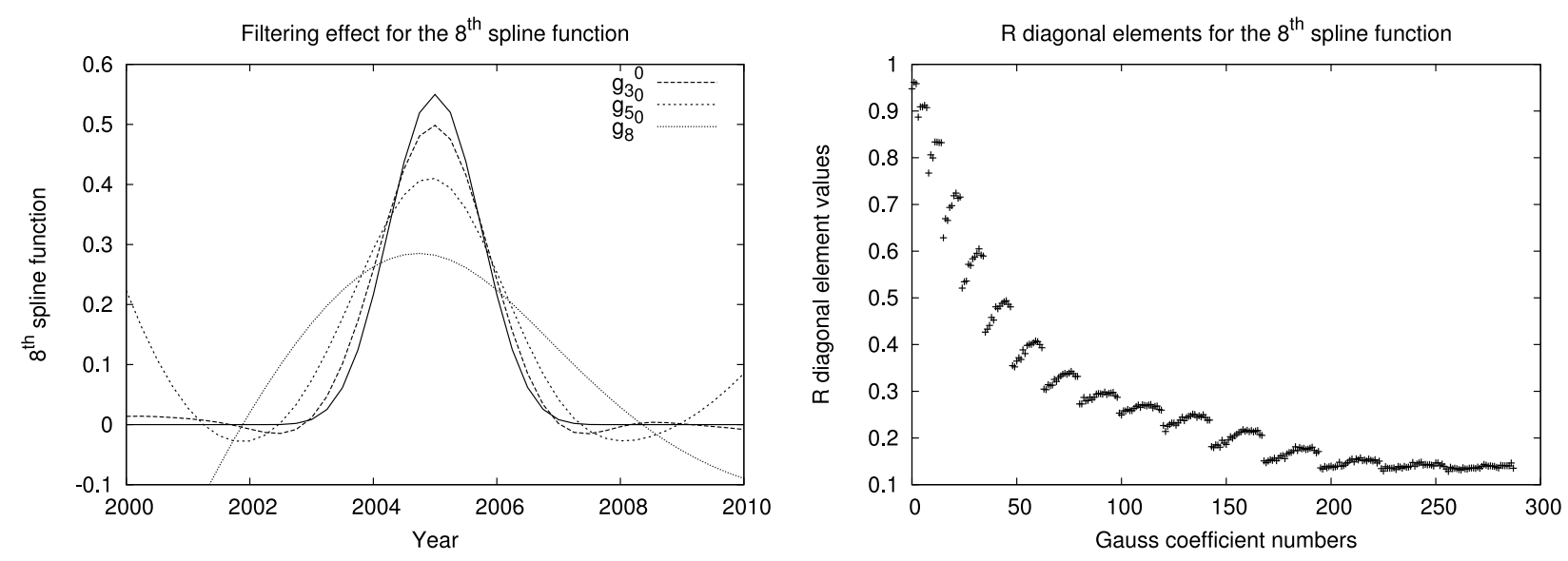

Fig. 8. Resolution analysis: On the left is drawn $8^{\text {th }}$ spline function (solid line) and the resulting filtered function for $g_{3}^{0}$, $g_{5}^{0}$ and $g_{8}^{0}$. On the right, diagonal element values of the resolution matrix given for the core field Gauss coefficients corresponding to the $8^{\text {th }}$ spline function are plotted. The Gauss coefficients are numbered in lexicographic order.

and the obtained Gauss coefficient temporal behaviors are strongly averaged over time. This result is consistent with the observed SA power spectrum decreases in Fig. 2. The right panel of Fig. 8 displays the diagonal elements of the resolution matrix extracted for the core field Gauss coefficients associated with the $8^{\text {th }}$ spline function. We checked that for our temporally un-constrained model, these diagonal elements are all 1. For the regularized model the diagonal elements rapidly decrease with the $\mathrm{SH}$ degree. The dependence on the SH order is limited. Although the effect of regularization is small at low $\mathrm{SH}$ degree, it is already present. However, it certainly does not affect significantly our candidate models to the IGRF.

\section{Conclusion}

We have presented the second generation of the GFZ Reference Internal Magnetic Model (GRIMM-2). As for the first generation, the model has been derived to provide an accurate description of the core field, its temporal behavior and in particular of the secular acceleration. The data set used covers the years from 2001.0 to 2009.5. The core field model is reasonably accurate over the data time span. The B-spline functions used allow the calculation of field values from 2000 to 2011, however, users should be particularly careful when extrapolating the model outside the time interval $[2001: 2009.5]$. We have seen that the secular variation model is controlled by the regularization applied for the first and last year of the model, i.e. it should be used with caution outside the time interval [2002:2008.5]. Above SH degrees 12 or 13 the power spectra of the secular variation model diverges and regularization is required. As an example, a regularization can follow the tapering approach applied in Wardinski et al. (2008) to obtain estimates of spatially controlled small scale SV.

Regarding the secular acceleration we see that it evolves rapidly and reaches absolute values as large as $25 \mathrm{nT} / \mathrm{y}^{2}$. There is no evidence of repetitive patterns, strengthening or weakening of the acceleration. However, the regularization applied affects the acceleration as early as SH degree 4 or 5 , and some work is still required before one can downward continue the acceleration model at the core mantle bound- ary.

This model has been used as the parent model for the GFZ candidate to the $11^{\text {th }}$ version of the IGRF. The model can be downloaded together with some FORTRAN 95 softwares at http: / / www.gf z-potsdam.de/magmodels.

Acknowledgments. We would like to acknowledge the work of CHAMP satellite processing team and of the scientist working in magnetic observatories. We particularly thanks R. Holme and an anonymous reviewer for their constructive comments. IW was supported by the European commission under contract No. 026670 (EC research project MAGFLOTOM).

\section{References}

Lesur, V., I. Wardinski, M. Rother, and M. Mandea, GRIMM-The GFZ Reference Internal Magnetic Model based on vector satellite and observatory data, Geophys. J. Int., 173, doi:10.1111/j.1365246X.2008.03724.x, 2008.

Lowes, F. J. and N. Olsen, A more realistic estimate of the variances and systematic errors in spherical harmonic geomagnetic field models, Geophys. J. Int., 157, 1027-1044, 2004.

Maus, S., M. Rother, C. Stolle, W. Mai, S.-C. Choi, H. Lühr, D. Cooke, and C. Roth, Third generation of the Potsdam Magnetic Model of the Earth (POMME), Geochem. Geophys. Geosyst., 7, Q07008, doi:10.1029GC001,269, 2006.

Olsen, N., R. Haagmans, T. J. Sabaka, A. Kuvshinov, S. Maus, M. E. Purucker, M. Rother, V. Lesur, and M. Mandea, The Swarm End-toEnd mission simulator study: Separation of the various contributions to the Earth's magnetic field using synthetic data, Earth Planets Space, $\mathbf{5 8}$, 359-370, 2006a.

Olsen, N., H. Lühr, T. Sabaka, M. Mandea, M. Rother, L. Tøffner-Clausen, and S. Choi, CHAOS-A model of the Earth's magnetic field derived from CHAMP, Oersted, and SAC-C magnetic satellite data, Geophys. J. Int., 166(1), 67-75, doi:10.1111/j.1365-246X.2006.02959.x, 2006b.

Olsen, N., M. Mandea, T. J. Sabaka, and L. Tøffner-Clausen, CHAOS-2 - a geomagnetic field model derived from one decade of continuous satellite data, Geophys. J. Int., 179(3), 1477-1487, doi:10.1111/j.1365246X.2009.04386.x, 2009.

Reigber, C., H. Lühr, P. Schwintzer, and J. Wickert (Eds.), Earth Observation with CHAMP Results from Three Years in Orbit, Springer-Verlag, 2005.

Thomson, A. and V. Lesur, An improved geomagnetic data selection algorithm for global geomagnetic field modelling, Geophys. J. Int., 169, 951-963, doi: 10.1111/j.1365-246X.2007.03354.x, 2007.

Wardinski, I., R. Holme, S. Asari, and M. Mandea, The 2003 geomagnetic jerk and its relation to the core surface flows, Earth Planet. Sci. Lett., 267, 468-481, doi:10.1016/j.eps12007.12.008, 2008.

V. Lesur (e-mail: lesur@gfz-potsdam.de), I. Wardinski, M. Hamoudi, and M. Rother 